Sociologie et sociétés

\title{
Controverses sur la réactualisation du travail informel au Brésil
}

\author{
L'impact des mobilisations dans l'économie solidaire sur les
} conceptions du changement social et les stratégies syndicales et politiques

\author{
Controversies around the new surge of informal work in Brazil \\ The impact of solidarity economy sector molbilizations on the \\ vision of social change and union and political strategies
}

\section{Carole Yerochewski}

Volume 47, numéro 1, printemps 2015

Travail et informalité : nouvelles figures de l'exploitation et des mobilisations au nord et au sud

Work and the informal sector: new figures of exploitation and mobilisations in the north and south

URI : https://id.erudit.org/iderudit/1034424ar

DOI : https://doi.org/10.7202/1034424ar

Aller au sommaire du numéro

Éditeur(s)

Les Presses de l’Université de Montréal

ISSN

0038-030X (imprimé)

1492-1375 (numérique)

Découvrir la revue

Citer cet article

Yerochewski, C. (2015). Controverses sur la réactualisation du travail informel au Brésil : l'impact des mobilisations dans l'économie solidaire sur les conceptions du changement social et les stratégies syndicales et politiques. Sociologie et sociétés, 47(1), 201-224. https://doi.org/10.7202/1034424ar
Résumé de l'article

Rassemblant au Brésil toute une partie des travailleurs informels, l'économie solidaire est un sujet controversé parmi les acteurs syndicaux et politiques. Est-elle un lieu d'insertion des travailleurs, et (surtout) travailleuses, pauvres ou informels qui sont rejetés par un marché nécessairement compétitif ? Ou les différentes mobilisations, et certaines des pratiques autogestionnaires de ces travailleurs dits marginalisés, constituent-elles des luttes pour l'égalité et la justice sociale qui dépassent les revendications (en termes de salaires, de durée du travail, etc.) issues du compromis de la société salariale — compromis qui n’a jamais eu vocation à couvrir plus qu'une minorité de travailleurs dans le monde ?Étayé par des entretiens approfondis menés auprès de responsables communautaires, syndicaux et politiques impliqués dans l'économie solidaire au Brésil, l'article met ainsi en lumière les relations entre leurs choix stratégiques et les façons d'analyser le problème de la remontée du travail informel. Les controverses suscitées sont révélatrices de conceptions plus globales sur l'économie et la place des mobilisations de travailleurs dans le changement social.
Tous droits réservés ㄷ Les Presses de l’Université de Montréal, 2015

Ce document est protégé par la loi sur le droit d'auteur. L'utilisation des services d'Érudit (y compris la reproduction) est assujettie à sa politique d'utilisation que vous pouvez consulter en ligne.

https://apropos.erudit.org/fr/usagers/politique-dutilisation/ 


\title{
Controverses sur la réactualisation du travail informel au Brésil
}

\author{
L'impact des mobilisations dans l'économie solidaire \\ sur les conceptions du changement social \\ et les stratégies syndicales et politiques
}

\section{CAROLE YEROCHEWSKI}

Chercheure associée au CRIMT et au GIREPS

Courriel: carole.yerochewski@umontreal.ca

\begin{abstract}
A LORS QUE LES RÉFORMES Organisant la collecte sélective et le recyclage ont souvent abouti à exclure de ce marché, devenu rentable, celles et ceux qui les avaient pris en charge pour survivre, au Brésil, le gouvernement pétiste (de PT, Parti des travailleurs) de l'État du Rio Grande do Sul a adopté, en 2011, une politique de soutien à l'industrialisation du recyclage du $\mathrm{PET}^{1}$ qui a remis en cause l'affectation inégalitaire des surplus. La transformation du PET en flocons puis en fibres synthétiques, activité à plus forte intensité capitalistique que la collecte et le tri manuel des matériaux, ne raflera donc pas les surplus générés, au motif que, génératrice de profits, elle représenterait des activités à plus forte valeur ajoutée, c'est-à-dire produisant plus de richesses (mesurables par le traditionnel étalon qu'est le PIB). Mais associer gains de productivité, valeur et profits est une convention, qui repose sur la marchandisation du travail. Elle n'est pas le résultat naturel de la division technique du travail mais le produit des formes sociales de division du travail dominantes dans l'organisation capitaliste de la
\end{abstract}

1. Le PET est le plastique le plus utilisé pour fabriquer des récipients de liquide, et donc notamment les bouteilles d'eau. 
production $^{2}$. C'est donc cette convention que remet en cause la politique publique du gouvernement pétiste du Rio Grande do Sul.

Qualifiée à juste titre de «bidirectionnelle», cette «chaîne productive ${ }^{3}$ » du PET reconnaît la valeur de l'apport des catadores (les récupérateurs de matériaux recyclables $)^{4}$ : leur niveau de revenu a ainsi quadruplé pour atteindre 1200 reais par mois, ce qui les place au-dessus du salaire minimum (à 724 reais en 2014). À l'été 2014, 200 coopératives organisant 9000 catadores (40 000 à terme) s'occupaient de collecter, trier et presser en ballots les bouteilles de PET qui sont ensuite transformées pour servir notamment à fabriquer des sacs et des habits.

Quoiqu'il s'agisse d'une politique publique ${ }^{5}$ soutenue par des investissements importants (de l'ordre de 7 millions de reais), l'initiative n'a pas été conçue par le PT, mais vient de l'économie solidaire. Elle s'inspire directement d'une de ses success stories connues sous le nom de Justa Trama: il s'agit de l'association, depuis 2002, de plusieurs coopératives, du nord au sud du Brésil, qui produisent du coton écologique et des semences de la forêt amazonienne pour les boutons des habits, fabriquent les fils et tissus, confectionnent et commercialisent les vêtements en se répartissant les surplus selon les mêmes principes égalitaires entre les différentes coopératives parties prenantes. Entre 2011 et 2014, la coordonnatrice d'Univens, une des coopératives membres de Justa Trama située à Porto Alegre, était la directrice de l'économie solidaire au gouvernement pétiste du Rio Grande do Sul. Ancienne syndicaliste à la CUT (Central Unica dos Trabalhadores) ${ }^{6}$, elle a été l'une des militantes les plus impliquées dans la création du mouvement de l'économie solidaire.

2. Pour une vision approfondie de la façon dont la mesure de la productivité et l'évaluation des qualifications ne sont pas des résultats de la division technique du travail mais le produit de conventions et d'un jugement social sur les valeurs accordées aux différentes contributions, voir Stroobants (1993) et Gadrey (2002) sur le caractère historiquement et socialement variable de ces conventions. On peut aussi l'illustrer en reprenant l'exemple donné par Delphy (2001) sur la façon dont est catégorisé comme non productif le travail (gratuit) des femmes au foyer alors que les mêmes tâches effectuées dans un restaurant ou par une entreprise de nettoyage sont comptabilisées dans le PIB.

3. Nom donné par l'économie solidaire brésilienne (cadeia productiva).

4. Les catadores ont commencé à s'organiser à partir des années 1990 en coopératives avec l'appui d'organisations communautaires et de réseaux d'Église. Le Mouvement national des catadores de matériaux recyclables (MNCR) a été créé à partir d'une rencontre nationale en 1999 qui a rassemblé 1700 catadores. Le MNCR se bat pour faire reconnaitre le rôle des catadores et pour inscrire la lutte dans la préservation de la nature, contre la "privatisation» des déchets, "pour une vie digne (...) pour le droit à travailler honnêtement» et selon des principes solidaires comme l'«auto-organisation», la «démocratie directe», l' «action collective de support mutuel», la «solidarité de classe» (documents MNCR et Street Net). Leur mobilisation — toujours importante — leur a permis de voir adopter trois principaux décrets et lois entre 2006 et 2010 incitant les municipalités, par des règlements et des financements, à s'appuyer sur eux pour organiser la collecte sélective et le recyclage.

5. Depuis le changement de majorité politique intervenu aux élections d'octobre 2014 (pour la période 2015-2018), cette politique est actuellement suspendue sur l'État du Rio Grande do Sul. Mais elle a été construite avec l'appui du gouvernement fédéral et en collaboration avec le gouvernement uruguayen et les villes qui s'appuient sur les catadores et leurs coopératives pour effectuer la collecte et le tri.

6. La CUT, d'où vient l'ancien président Lula, ex-métallo dans la région industrielle de l'ABC, est la principale confédération syndicale brésilienne et la seule à s'être dotée (à la fin des années 1990) d'une politique pour organiser les travailleurs informels, via l'économie solidaire. 
On ne saurait toutefois réduire l'impact de ce mouvement sur les politiques publiques à des rencontres individuelles favorisées par l'appartenance à des réseaux de connaissance, sans questionner comment ces rencontres et ces réseaux ont pu se construire — à moins de les prendre pour des mécanismes «déjà là7 » (et d'ignorer qu'il existe d'autres types de success stories dans des États brésiliens gouvernés par des partis de droite).

L'objet de cet article est de montrer en quoi ces politiques reposent sur des définitions partagées de ce que peut être l'économie solidaire et, surtout, de ce que sont les problèmes ou «les dominations » à combattre - pour reprendre Armstrong et Bernstein (2009) —, et comment ces lectures se construisent en étant intimement liées avec les analyses sur le travail informel.

L'originalité de cet article tient donc dans le fait de mettre au jour les relations entre les façons d'analyser les enjeux de la recrudescence du travail informel et les stratégies poursuivies, en particulier par les acteurs politiques et syndicaux. À ma connaissance, les différents travaux hétérodoxes sur l'économie informelle ou sur les mobilisations de travailleurs informels n'ont pas approfondi ces relations. On peut en effet classer ces travaux en deux segments distincts.

Une partie des travaux critiques de ceux présentant l'économie informelle comme un secteur autonome ont mis en lumière son imbrication avec l'économie formelle (Fernandez-Kelly, 2006; Lautier, 2006; Portes et al., 1989, Agarwala, 2009), et ce, sous diverses formes pas nécessairement illégales: des entreprises souvent petites ou des coopératives qui ne sont pas enregistrées comme telles ou qui ne déclarent pas leurs salariés ou membres, travaillent pour des entreprises formelles qui, elles aussi, peuvent ne pas déclarer une partie des salariés (recrutés notamment à l'aide de contrats temporaires) ou recourir directement, ou par l'entremise d'autres entreprises sous-traitantes, à des travailleurs à domicile; eux-mêmes susceptibles de mobiliser leurs relations familiales ou de voisinage pour assurer la livraison, dans les délais, de commandes imprévisibles en volume et en temps, comme cela a cours notamment dans la confection et les industries saisonnières. On voit aussi, par exemple dans les petites rues de cette mégalopole qu'est São Paulo, des vendeurs ambulants placer non plus des objets de pacotille mais des cartes de crédit pour des banques qui les contractent comme travailleurs indépendants et les paient à la commission. Ainsi, dans le cadre des processus de flexibilisation du travail et de l'emploi, et de réduction des protections sociales acquises, de nouvelles formes de travail informel font leur apparition tandis que le contenu d'anciennes activités (vente ambulante, travail à domicile) est «réactualisé» (Leite, 2009a). Ces travaux (notamment Portes et al., 1989; FernandezKelly, 2006) montrent aussi l'imbrication des rapports sociaux de classes, de sexes et de races (Kergoat, 2005), qui contribue à ce que l'on retrouve plus souvent dans le travail informel - et dans ses activités les plus exploitées, qui offrent moins de

7. Voir à ce sujet les critiques au courant d'analyse des mouvements sociaux dits du processus politique par Goodwin et Jaspers (2004). 
barrières à l'entrée - certaines catégories de la population, telles les femmes ayant des enfants encore petits dans le travail à domicile. Dans tous les cas, les auteurs soulignent le rôle joué par l'État dans les re-régulations de l'emploi et l'informalisation de l'économie et des relations de travail, tant au Sud qu'au Nord dorénavant (Portes et al., 1989, Lesemann, 2015), dévoilant ainsi l'inanité des discours des institutions internationales qui proposent comme remède à la pauvreté de formaliser l'informel (Lautier, 2006).

Toutefois, tout en relevant que le développement de l'informalité doit nous conduire à revoir les cadres d'analyse issus de la société salariale (Brenner, 1999; Lesemann, 2015), ce pan hétérodoxe de littérature ne va guère au-delà du fait de constater que l'informalisation grandissante affaiblit le syndicalisme et «altère les modèles de lutte et de relations de classes» (Portes et al., 1989: 308), confrontant les travailleurs organisés à un défi qui «jusqu'ici, n’a pas été relevé» (ibid.). Ils n'approfondissent pas comment les travailleurs informels arrivent à s'organiser ni quels types de luttes ils mènent — si ce n'est sous l'angle d'une critique des visions idéalisées d'une économie informelle solidaire, que reprend la Banque mondiale, qui la présente comme un lieu de débrouillardise des pauvres (Lautier, 2003 et 2006) et des «entrepreneurs aux pieds nus » (expression de l'idéologue libéral Guy Sorman).

Pour voir explorées les luttes de ces travailleuses et travailleurs informels, comment elles innovent en matière de répertoires d'action collective et s'emparent de «leviers symboliques» pour faire connaître leur cause (Chun, 2009), il faut aller examiner un autre segment hétérodoxe; celui-ci critique les théorisations qui assimilent de facto économie informelle à travailleurs inorganisés et économie formelle à travailleurs organisés (Agarwala, 2009: 34) et nient la capacité des travailleurs informels à mener des actions collectives contestatrices de l'ordre social (Lindell, 2010). Or, avec l'appui d'organismes communautaires et de syndicats, mais plus souvent en position conflictuelle avec ces derniers (Lindell, 2011; Croucher, 2007), ils et elles construisent des solidarités non seulement locales mais transnationales (Lindell, 2011), pouvant déboucher sur des avancées importantes, comme en Inde où l'État mutualise des fonds pour financer une protection sociale et une éducation de base aux travailleurs et (surtout) travailleuses enregistrés auprès de leur association-syndicat (Agarwala, 2013). Cependant, ces travaux, tout en soulignant l'inertie des confédérations syndicales ou leurs stratégies susceptibles d'entraver la mobilisation de ces travailleurs (Chun, 2009), n’ont pas expliqué pourquoi elles ont une «analyse incorrecte» car «partielle» d'évènements, qui leur fait prendre des décisions contraires aux travailleurs (Bieler, 2014: 121).

C'est en revanche ce que l'article met en lumière: il montre en quoi les controverses suscitées par les mobilisations de travailleurs et par leurs redéfinitions des problèmes, ou dominations, reposent sur des perceptions qui s'écartent ou, au contraire, s'inscrivent dans des analyses naturalisées de l'économie informelle. La naturalisation opère lorsqu'on assimile l'économie informelle à une phase précapitaliste d'un développement économique devant l'absorber à terme. Ce type d'analyse 
véhicule l'idée — tant «orthodoxe» que «néo-marxiste» — d'une «incorporation progressive de la force de travail dans les relations modernes de travail» (Portes et al., 1989: 3 07). L'article met en évidence à ce titre la stratégie de la CUT dans l'économie solidaire, et les conflits qu'elle soulève, y compris au sein de ses propres rangs, quand la direction de la centrale s'enferre dans une défense de la société salariale revenant à exclure ceux qu'elle prétend dorénavant organiser.

En comparant ensuite les pratiques autogestionnaires associées aux positionnements dans les controverses, l'article montre comment ces analyses naturalisées reposent, corollairement, sur une conception du travail qui alimente un rapport hétéronome à celui-ci (ou aliéné comme Marx le qualifiait, sans toutefois remettre en cause les postulats sur le travail à la source de sa marchandisation ${ }^{8}$ ). La conclusion revient sur les principaux résultats.

\section{CADRE D'ANALYSE ET MÉTHODOLOGIE}

Pouvoir examiner le poids de certaines perceptions dans les stratégies, et donc le rôle des idées, et en l'occurrence des identités collectives, puisqu'il s'agit d'examiner le mouvement de l'économie solidaire, implique de se doter d'une conceptualisation qui n'établit aucun présupposé sur le contenu même de ces identités. Cela suppose aussi d'adopter une méthodologie d'observation permettant de vérifier l'impact sur les stratégies de ces idées — ou façons de définir les problèmes. Et vice-versa: il faut aussi pouvoir saisir l'impact des pratiques, et en particulier des modalités d'interaction, sur les représentations des problèmes, car identités et interactions sont interdépendantes (Yerochewski, 2015) et constituent des concepts clés de l'analyse des mouvements sociaux, comme le souligne la convergence sur ce point des différents courants (Diani, 2003; McAdam, 2003; Bernstein, 2008).

Cette recherche s'appuie sur une conceptualisation des identités collectives en matrice cognitive et normative (Surel, 1998), et donc à même de prescrire l'action. Une telle conceptualisation ne part d'aucun a priori sur le contenu et permet d'opérationnaliser la définition de l'identité collective proposée par Melucci ${ }^{9}$. Relever les orientations (partie normative) et la lecture des opportunités (partie cognitive), dans le champ délimité par les différentes controverses autour des problèmes publics, informent (explicitement ou implicitement) des façons d'identifier les (causes et d'imputer les responsabilités des) problèmes, ou dominations, contre lesquelles on se mobilise. Ceci est cohérent avec l'approche cognitive des politiques publiques qui a montré que les orientations ou solutions proposées dépendent de la façon de définir les problèmes (Jenson et al., 1999).

8. Pour une vision exhaustive des débats philosophiques sur le travail et une critique de l'utopie marxiste, voir Méda (1995).

9. «L'identité collective est une définition interactive et partagée produite par plusieurs individus (ou groupes à un niveau plus complexe) concernant les orientations de leur action et le champ d'opportunités et contraintes dans lequel une telle action doit avoir lieu» (Melucci, 1996: 70). Les soulignements sont de l'auteur. 
Les résultats présentés s'appuient essentiellement sur la cinquantaine d'entrevues approfondies menées au cours d'un séjour de plusieurs mois au Brésil, en 2008 (et en partie réactualisées lors d'un bref séjour de «retour» en 2012), avec des responsables de coopératives, d'organismes communautaires et de syndicats locaux et nationaux de la CUT ainsi que quelques militants du PT. Lors de l'analyse, les interprétations cognitives et normatives ont été distinguées en s'appuyant sur les adverbes et conjonctions de coordination ( «parce que», «car», ou bien «pour que» et «si... alors») ainsi que sur certains verbes (comme «devoir», «falloir») qui structurent le discours.

Par ailleurs, pour vérifier les correspondances entre identités et actions, il a été demandé aux interlocuteurs de préciser quelles étaient leurs références en termes notamment de pratiques autogestionnaires et d'indiquer, quand il s'agissait des interlocuteurs syndicaux, quelles étaient les stratégies adoptées, par rapport au positionnement exprimé sur les controverses. Cette partie des entretiens a été complétée par un recueil de divers documents permettant d'étayer des analyses en termes d'idéaux-types des pratiques autogestionnaires citées en référence.

Quant au choix du cas de l'économie solidaire, il se veut paradigmatique (Flyvbjerg, 2006). Il possède plusieurs qualités théoriques: tout d'abord, l'économie solidaire au Brésil mobilise les travailleurs au cœur des rapports sociaux de domination (Kergoat, 2009); et elle les organise au travers de multiples lieux d'interaction, par la mise en œuvre de coopératives et associations autogérées, par des rencontres à différents échelons et sur différents thèmes avec d'autres mouvements sociaux et différentes institutions; elle est à ce titre un «nouveau» mouvement de travailleurs, qui mobilise celles et ceux que les syndicats n'organisent habituellement pas. C'est aussi un lieu d'alliances entre les principales composantes de la société civile, dont la CUT. L'économie solidaire brésilienne offre ainsi un terrain fertile pour examiner pourquoi de nouvelles identités collectives de travailleurs émergent et comment les syndicats — et la CUT en particulier — se positionnent face à l'émergence de ces nouvelles identités/aspirations.

\section{LA REMONTÉE DU TRAVAIL INFORMEL AU BRÉSIL ET LES CONTROVERSES DANS L'ÉCONOMIE SOLIDAIRE}

Longtemps, le travail informel n'a pas été considéré comme un problème au Brésil. Cette situation est héritée de l'adoption en 1943 des lois consolidées du travail (Consolidação das leis do trabalho, CLT), qui ont fourni à la seule catégorie des travailleurs formels - détenteurs d'un livret de travail, ou carteira assinada, délivré par l'employeur — l'accès aux droits et à la syndicalisation, en contrepartie de la mise sous tutelle des syndicats par l'État. Il s'agissait de pousser le Brésil dans les voies du capitalisme moderne en régulant l'exploitation d'une main-d'œuvre dans les concentrations industrielles naissantes, sans remettre en cause les concentrations de pouvoir politique et économique de l'oligarchie brésilienne. Ceci a débouché sur un encadrement arbitraire, clientéliste et concurrentiel des différents segments du marché du travail (Paoli, 2000), tandis que les activités et les modalités de (sur) vie des travailleurs 
informels ont été rendues invisibles, masquant de surcroît l'ampleur des discriminations de genre, ou raciales et liées à la tradition esclavagiste du Brésil.

Cette situation a été tolérée tant que le marché du travail formel semblait régulièrement absorber des travailleurs informels. En 1980, la part des salariés avait fortement progressé et atteignait $66 \%$ de la population économiquement active; parmi eux, $60 \%$ étaient des travailleurs formels (Ramalho, 1999, p. 162), entretenant ainsi l'idée d'une lente extension de la société salariale, un paradigme alors dominant malgré son incomplétude au Brésil (Ramalho et Santana, 2003; Ferraz, 2005).

Le travail informel va cesser d'être considéré comme un épiphénomène avec l'éclatement des crises économiques dans les années 1980 et 1990, conséquences des politiques suivies par les militaires après le coup d'État de 1964, des réformes orthodoxes demandées par le FMI et de l'ouverture brusque du pays à la concurrence par les gouvernements néolibéraux (élus après la démocratisation du pays et l'adoption de la Constitution de 1988), ce qui entraîne de nombreuses faillites industrielles. Le taux de chômage des travailleurs formels explose — il dépasse les $20 \%$ au début des années 2000 dans plusieurs des grands centres urbains du Brésil —, tandis que les flux entre travail formel et informel s'inversent, du fait qu'une partie des emplois formels s'informalisent (Leite, 2009a) : le taux de travailleurs informels remonte au point de redépasser celui des travailleurs formels.

Débats et controverses éclatent alors au sein des universitaires et des acteurs politiques et sociaux, d'autant plus que ce n'est pas seulement la remontée du travail informel qui invite à se demander s'il s'agit là non d'un phénomène conjoncturel (Leite, 2009a), mais d'une réorganisation globale des activités (Portes et al., 1989); les mobilisations de travailleurs informels dans l'économie solidaire, tout comme celles d'ailleurs des travailleurs ruraux et des agriculteurs familiaux ${ }^{10}$, amènent des relectures de ce qui constitue le développement économique et la transformation sociale.

\subsection{Un mouvement social articulant lutte contre la pauvreté et démocratisation}

L'idée que la crise n'est pas conjoncturelle mais structurelle et remet en cause un modèle de développement particulièrement inégalitaire et excluant au Brésil se manifeste dans les politiques adoptées dès 1996 par la municipalité de Porto Alegre. La mairie, alors conduite par l'équipe de Tarso Genro ${ }^{11}$, crée une supervision de l'économie solidaire au sein de son secrétariat municipal à l'industrie et au commerce, signi-

10. Ils se sont organisés massivement en coopératives, à partir de la seconde moitié des années 1980, avec le Mouvement des travailleuses et travailleurs sans terre, MST, et des syndicats ruraux, en général membres de la CUT.

11. À la suite d'un accord entre, d'une part, le courant trotskyste majoritaire au sein du parti des Travailleurs du Rio Grande do Sul dirigé par Olivio Dutra et, d'autre part, le courant majoritaire au sein du PT national mais minoritaire dans l'État de Rio Grande do Sul, et représenté par Tarso Genro, il y a alternance des candidats entre les deux courants du PT, lors des élections sur Porto Alegre ou sur le plan de l'État. Cette précision est importante car si Olivio Dutra est bien connu pour avoir créé le budget participatif de Porto Alegre, après son élection en 1988, et accueilli le $1^{\text {er }}$ FSM à Porto Alegre en 2001, quand il était gouverneur du RS (entre 1998 et 2002), sa politique dans l'économie solidaire a été contestée par tous ses 
fiant ainsi qu'elle considère ces initiatives de création collective de travail et revenu comme partie prenante d'une redéfinition du développement économique — et non comme une politique sociale d'inclusion qui ne remet pas en cause ses contours (Sarria Icaza, 2008). Un incubateur de coopératives populaires et une banque populaire destinée à soutenir les initiatives des travailleurs informels sont aussi créés et mis en œuvre de façon à "établir un dialogue en condition symbolique d'égalité» (voir Barcellos et Beltrão, 2000, p. 172-174-176-177). Quoiqu'ils ne soient toujours pas organisés ni représentés d'une manière significative par les syndicats (Georges, 2011), alors que la Constitution adoptée en 1988 en a ouvert la possibilité, les travailleurs informels sont ainsi reconnus dès 1996 comme des sujets collectifs par la municipalité de Porto Alegre - une situation qui se retrouvera dans d'autres villes ayant des budgets participatifs particulièrement intégrateurs des habitants populaires (Souza, 2009).

Deux principaux facteurs ont contribué à l'adoption de ces politiques, qui ont fait école auprès d'autres municipalités du Rio Grande do Sul. Il y a, d'une part, les rencontres organisées par Caritas, un réseau d’Église s’inspirant de la théologie de la Libération et des préceptes d'éducation populaire de Paulo Freire, très actif dans le soutien aux initiatives des travailleurs informels et du MST. Il a appuyé la première coopérative de catadores à Belo Horizonte. Sur le Rio Grande do Sul, il a confié des recherches-actions à L. I. Gaiger (2000), l'un des universitaires les plus impliqués et reconnus au Brésil dans ce domaine (Lechat, 2004). Elles ont alimenté (dès 1995-1996) les réflexions sur les initiatives collectives de création de travail et revenu (geração de trabalho e renda) avec la municipalité de Porto Alegre et d'autres acteurs de la société civile, dont la CUT régionale, et ont permis d'identifier des «repères fondamentaux» concernant tant la signification des expériences que les politiques publiques appropriées pour les soutenir (Sarria Icaza, 2005; Lechat, 2004).

D'autre part, les politiques adoptées par Porto Alegre répondent aussi aux demandes qui émanent du budget participatif. Celui-ci constitue à cette époque (les années 1990) une référence exemplaire en termes d'implication et de participation massive des habitants des quartiers populaires, qui peuvent y faire valoir leurs visions des priorités et du bien commun dans leurs propres termes (Baïocchi, 2003). Le budget participatif a lui-même suscité des coopératives: Univens a ainsi été créée par un groupe de femmes qui a pensé, en 1996, que «cela apporterait une réponse au besoin de travailler et en même temps, ce serait une action participative et collective» (entretien 2008). C'est l'une de ces femmes qui a été directrice de l'économie solidaire dans le RS entre 2011 et 2014.

Peu de travaux ont souligné à quel point la démocratisation au Brésil, emmenée par les mobilisations de la société civile, «a fortifié dans le mouvement social brésilien un processus de discussion dirigé vers la question de la démocratisation dans le monde du travail» (Leite, 2009b, p. 42). Grâce à une campagne menée par un organisme très 
reconnu - l'Action de la citoyenneté contre la faim, la misère et pour une vie digne, auquel participaient plusieurs acteurs de la société civile —, de multiples comités locaux vont appuyer la création de qui est appelé alors des alternatives de travail et revenu, car s'insérant dans une autre vision que celle développée par les institutions internationales. Cette campagne, qui a pris appui sur la mobilisation de masse en faveur de la destitution du premier ministre Collor (en 1994), a débouché sur le plus important mouvement social «depuis la lutte pour les droits démocratiques en 1985 » (Singer, 2002, p. 119).

L'économie solidaire est aussi nourrie par les actions de travailleurs formels qui, à la suite de la faillite de leur entreprise — phénomène répandu dans les années 1990 —, récupèrent avec l'appui de syndicalistes de la CUT, et souvent à leur initiative, leur entreprise. «Les travailleurs de diverses branches d'activité ont entamé la formation de coopératives, les mouvements sociaux se sont mis à promouvoir les pratiques d'autogestion, les universités et autres entités ont commencé à appuyer la création des empreendimentos ${ }^{12}$ solidaires ${ }^{13}$.»

À la différence des politiques des institutions mondiales, les initiatives de l'économie solidaire au Brésil s'inscrivent donc dans un mouvement social de travailleurs, qui articule la lutte contre la pauvreté et l'accès au registre des droits (versus le clientélisme) et à la citoyenneté participative. Au fur et à mesure de son essor, ce mouvement social va en outre faire émerger une autre notion de la transformation sociale que celle, typique, de la société salariale-fordiste, qui prévoit (en résumé) le partage des gains de productivité contre l'abandon au patronat de l'organisation du travail et des choix de production.

Les enjeux de protection de l'environnement, d'égalité entre les hommes et les femmes, et entre les différentes ethnies, sont aussi pris en compte et les différentes aspirations s'articulent dans des pratiques autogestionnaires de coopératives et d'associations gérées collectivement: celles-ci relient les choix de production à la satisfaction de besoins sociaux discutés collectivement, au sein et au-delà des coopératives et groupes autogérés.

Univens peut fournir un exemple. La coopérative a été à l'initiative de la création d'une crèche qui ne répond pas qu'aux besoins de garde de ses membres, mais aussi du quartier. Il ne s'agit donc pas seulement de concilier travail et famille pour les coopératrices concernées. Comme d'autres exemples de coopératives qui vont mettre en lumière des enjeux dits locaux (telle la nécessité de fournir une alimentation de qualité aux écoliers, en s'appuyant sur les coopératives d'agriculteurs familiaux), Univens rend visibles des besoins et aspirations au-delà du lieu de production marchande, qui influencent et justifient en retour les choix de production et d'organisation. Ainsi, alors qu'Univens (avec les autres coopératives du réseau Justa Trama) a créé une

12. Empreendimento n'a pas d'équivalent en français, et se réfère au fait d'entreprendre collectivement.

13. Pereira, Maria C. (2007: 18), Experiências auto-gestionárias no Brasil e na Argentina. [Dissertação de mestrado], Campinas, Faculdade de Educação, Unicamp, cité par Leite, 2009b: 42. 
marque et confectionne des habits de qualité, elle a choisi de maintenir un prix de vente modéré des vêtements afin de favoriser l'accès à des familles peu aisées. Ceci se répercute sur les revenus que leur activité peut générer. Mais c'est un choix collectif effectué en assemblée générale: "Tout le monde décide de la production, du prix qu'on va demander, et ainsi tout le monde sait combien il va gagner pour ce mois» (entretien, 2008).

Univens propose ainsi une autre compréhension de la performance, en rendant interdépendants les choix et finalités de la production marchande, l'organisation du travail au sein de la coopérative et d'autres besoins et activités, rémunérées ou non, qui sont habituellement dominées et donc dévalorisées. L'exemple illustre comment l'économie solidaire, du moins une partie d'elle, remet en cause la prédominance de la marchandisation, propre à la logique capitaliste, sur l'organisation sociale. À Univens, le travail n'est plus seulement le moyen de produire des richesses, selon la conception étroite promue par Adam Smith, qui revient à exclure tout le travail dit de reproduction sociale alors que celui-ci est aussi essentiel à la production du «vivre- ensemble» (Galerand et Kergoat, 2014) ${ }^{14}$.

\subsection{Le maintien d'analyses orthodoxes de l'économie informelle}

Mais tous les protagonistes (organisations de la société civile, dont la CUT, universitaires, agents de développement, militants du PT, etc.), qui se sont retrouvés, dans la foulée du premier Forum social mondial à Porto Alegre en 2001, pour construire le Forum brésilien de l'économie solidaire (FBES), ne partagent pas la même définition des problèmes. Au-delà d'afficher une orientation commune pour l'économie solidaire, ils n'analysent pas de la même façon les facteurs de crise et de remontée du travail informel ni, du coup, le potentiel que représentent l'économie solidaire et la mobilisation des travailleuses et travailleurs.

Pour une partie des universitaires et des syndicats de la CUT, dont le syndicat des métallos de la région industrielle de l'ABC qui donne le «la» à la CUT, c'est-à-dire son orientation majoritaire, y compris dans l'économie solidaire (conseiller syndical, 2008), la crise des années 1990 est la conséquence, décalée par rapport à d'autres pays, des mutations des technologies et du capitalisme. S'ils remettent en cause le capitalisme dans sa capacité à assurer une société socialement juste, ces syndicalistes ne s'interrogent pas sur ses fondements. Il s'agit seulement d'en corriger les effets néfastes pour les travailleurs «exclus du processus productif pour des raisons d'âge, de scolarité, de problèmes de santé ou de séquelles liés aux accidents de travail» (résolution du $3^{\mathrm{e}}$ congrès du syndicat des métallos de l'ABC) ${ }^{15}$. L'économie solidaire peut devenir

14. À noter qu'avec les restructurations du capitalisme, de plus en plus d'activités essentielles à la compétitivité des cadres des villes globalisées sont déclassées dans la sphère dite de reproduction sociale, considérée «improductive» (cf. tout le travail informel des traiteurs, livreurs, petites entreprises de confection ou de sous-traitance logicielle). Voir notamment les ouvrages de Saskia Sassen.

15. Résolutions du $3^{\mathrm{e}}$ congrès (1999) do Sindicato dos Metalúrgicos do ABC, en ligne: www.abcdeluta.org.br/materia.asp? id_CON=2326 (document intitulé: Prioridades para o próximo periodo). 
une béquille pour l'extension de la société salariale en servant à inclure dans un marché par nature compétitif les rejetés de la modernisation. Ceci à condition d'y développer de grandes coopératives compétitives, susceptibles de fournir l'équivalent des droits des travailleurs formels aux membres des coopératives. Sinon, on participe à la flexibilisation du marché du travail. En 1999, le syndicat des métallos de l'ABC a donc adopté une résolution, reprise un an plus tard par la CUT, qui soutient la création de grandes coopératives apportant «la croissance économique aux secteurs qui se sont montrés non viables» (idem).

La logique peu explicite de cette résolution est sous-tendue par les analyses d'un économiste péruvien marxisant, Anibal Quijano ${ }^{16}$, qui est une référence pour la CUT (Ferraz, 2005 : 138). Pour Quijano, l'économie solidaire dite populaire, celle qui renvoie en fait aux initiatives souvent petites des travailleurs informels, construites à partir de leur lieu de vie, est limitée par nature à être une économie de subsistance parce qu'elle reposerait sur des liens, familiaux ou de voisinage, dits primaires. Au contraire, les coopératives sont "potentiellement viables, car capables de couvrir des branches ou des secteurs entiers du système économique» parce qu'elles établissent «des relations avec les marchés locaux, nationaux ou globaux» (Ferraz, 2005: 138).

Les positions de Quijano ne se différencient pas des analyses néoclassiques dualistes sur l'économie informelle, sauf par le fait de considérer que le travail informel est marginal (voir Lautier, 2013: 202), c'est-à-dire que ses travailleurs ont été laissés en plan par le développement et que cette situation ne se résorbera pas d'elle-même. C’est pourquoi il faut agir. Mais en ignorant que les «formes non capitalistes de production et de travail étaient fonctionnelles avec la reproduction du capital», comme le souligne Guimarães (2011:6) en critiquant la vision dualiste de Quijano, la stratégie préconisée perpétue une vision étapiste qui naturalise le développement économique capitaliste, en faisant un passage obligé car «libérant» le travailleur des liens primaires de la féodalité pour mieux développer les forces productives (et donc la croissance).

\subsection{Controverses et stratégies de lutte contre les fausses coopératives}

Un groupe d'universitaires réunis par la fondation Unitrabalho, qui a été créée à la mi-1990 par des professeurs et recteurs proches ou membres de la CUT, va alimenter ces controverses et chercher à bannir de l'économie solidaire les initiatives soutenues par Caritas, qui s'appuient, pour eux, sur des liens dits primaires et donc sur des formes précapitalistes de développement et à l'économie informelle. Qualifiées d'économie de pauvres, ces initiatives ne pourraient être qu'au service d'une logique d'assistance en perpétuant un «aspect de subsistance» (Universitaire Unitrabalho, 2008).

Pour organiser les pauvres, un responsable d'Unitrabalho préconise de structurer l'économie solidaire en coopératives complexes (ou coopératives de coopératives) dans lesquelles les entreprises récupérées ont un «rôle très important»: il consiste à

16. Quijano, A. (2002), «Sistemas alternativo de produçao?», in B. de Sousa Santos (org.), Produzir para viver: os caminhos da produção não capitalista, Rio de Janeiro, Editora Civilização Brasileira. 
«mener l'économie solidaire» parce que leurs coopérateurs sont «déjà passés par la situation d'être salariés »; «d'une certaine façon», ils ont fait le choix de l'autogestion (entretien, 2008). Cette affirmation est en fait très contestable, comme on va le voir dans la partie 3.

Mais ce qu'il est important ici de souligner, c'est que les entreprises récupérées rassemblent les anciens travailleurs formels, qui étaient le plus souvent syndiqués. On retrouve ainsi dans les propos de ce responsable d'Unitrabalho une lecture orthodoxe du marxisme, selon laquelle la «classe ouvrière assume le rôle d'avant-garde et ses luttes sont fondamentales, tandis que celles de travailleurs qui n'ont pas été forgées dans la lutte syndicale ne peuvent amener que des combats secondaires » (Lechat, 2004: 201). En d'autres termes, Unitrabalho, tout comme le syndicat des métallos de l'ABC, continue de «s'attendre à ce que la lutte des classes, les confrontations, et les négociations procèdent tout au long de cette division de base» que constitue l'opposition entre une masse de salariés organisés et les dirigeants et propriétaires (Portes et al., 1989: 308).

Ces positionnements ont des conséquences qui dépassent le fait de ne pas porter assez d'attention aux travailleurs que les syndicats n'organisent pas habituellement: c'est la capacité même des travailleuses et des travailleurs à transformer le monde quand ils et elles se mobilisent qui est en fait dépréciée ou niée, voire brisée par les stratégies adoptées. Une autre controverse, en réalité corollaire, ayant trait aux stratégies de lutte contre les «fausses coopératives» va permettre de l'exposer.

Le terme de fausses coopératives désigne les coopératives suscitées par des entreprises, ou même par des États ou des municipalités (Lima, 2003), pour contourner le paiement des cotisations sociales et réduire le coût du travail. En effet, la loi brésilienne sur le coopérativisme (1971) prévoit que les coopérateurs ne peuvent avoir le statut de salariés (et vice-versa). Les coopérateurs se partagent donc les surplus, s’il y en a. Taillée pour encourager le regroupement à l'export des producteurs agricoles, la loi a été amendée en 1994 à la demande du MST, qui a voulu accélérer le développement des coopératives d'agriculteurs familiaux sans se heurter à la bureaucratie administrative: désormais, les coopératives sont a priori considérées «autonomes». Les entreprises ont tout de suite saisi l'opportunité de les utiliser pour contourner leurs responsabilités d'employeur.

La prolifération des fausses coopératives a bien sûr alimenté au sein de la CUT les réticences envers l'économie solidaire. Aussi, un projet de loi a été élaboré dans la première moitié des années 2000, à l'époque où le ministre du Travail est un ancien président du syndicat de l'ABC. Son principe consiste à contrôler le fonctionnement autogestionnaire et à rendre moins attractif le recours aux fausses coopératives. Pour ce faire, le projet prévoit que les coopérateurs bénéficient des mêmes conditions qu'un salarié et que la coopérative tienne des assemblées générales régulières.

Une vive opposition se manifeste à la $4^{\text {e }}$ plénière de l'économie solidaire, en 2008, contre ce projet et surtout contre l'article qui prévoit de sanctionner les coopératives ne fournissant pas à leurs membres un revenu équivalent au salaire de la convention 
collective ou tout du moins au salaire minimum. Car il donne un coup d'arrêt à l'existence de plusieurs petites coopératives ou de groupes au statut informel (parce qu'ils ne réunissent pas les 20 membres prévus par la loi de 1971 pour former une coopérative). Même si l'état des lieux réalisé en 2007 à la demande du secrétariat national à l'Économie solidaire signale une amélioration des niveaux de vie et des relations de travail, qui n'est pas étonnante à partir du moment où l'économie solidaire permet à des travailleurs informels de sortir de relations clientélistes et de mise en concurrence individuelle, nombre d'initiatives ne sont pas pour autant capables de fournir l'équivalent des droits des travailleurs formels aux coopérateurs membres: elles n'ont tout simplement pas un niveau d'activité permettant d'assurer toute l'année de tels revenus. Plusieurs n'arrivent donc même pas à fournir l'équivalent d'un salaire minimum sur l'année.

Fait marquant, une grande partie des membres d'Unisol, la structure de représentation des coopératives créée en 1999 à l'initiative du syndicat des métallos de l'ABC pour soutenir sa vision stratégique, s'opposent en 2008 au projet de loi. Il faut dire que depuis sa création, à partir du regroupement d'une douzaine d'entreprises récupérées dans l'État de Sao Paulo, Unisol a évolué d'une façon inattendue, avec l'entrée de nouveaux membres représentatifs de la diversité de l'économie solidaire au Brésil. Et ceux-ci risquent de pâtir directement d'un projet de loi jugé inacceptable parce qu'il va surtout briser des initiatives et des dynamiques collectives. Et aussi parce qu'il revient à mettre «sous tutelle de l'État les règles de l'autogestion», comme l'explique la coordonnatrice d'Univens, qui est à l'époque membre de l'exécutif d'Unisol. Pour elle, «la question est de savoir si nous croyons ou pas dans la capacité du travailleur».

Plusieurs opposants au projet de loi défendent une stratégie alternative, qui consiste justement à s'appuyer sur ces travailleuses et travailleurs des fausses coopératives: il s'agit de les aider à s'organiser pour oser prendre le pouvoir qui leur revient en transformant ces fausses coopératives en vraies. Un interlocuteur du syndicat des métallos de Porto Alegre, qui est opposé à l'orientation défendue par le syndicat des métallos de l'ABC, a fait l'expérience avec succès:

Je vais te raconter qu'ici [à Rolante], dans le Rio Grande do Sul, il y avait un groupe de femmes, mais deux personnes les ont enjôlées. Ces deux personnes ont monté une coopérative avec une assemblée, des signatures, etc. Elles gagnaient beaucoup d'argent sur le dos de ces femmes. Ce que nous avons fait, moi et [F.] ? Bon, on aurait pu les dénoncer au ministère public [...] ou nous pouvions entamer une lutte politique ${ }^{17}$. Nous avons parlé avec une femme qui disait que ces deux personnes gagnaient 3000 reais et elles $200[\ldots]$ «Pourquoi ne faites-vous pas une liste de signatures pour demander une assemblée générale?»; «Vous nous aidez?»; «Nous vous aidons». Les femmes ont fait une assemblée, ont expulsé les deux personnes de la coopérative et se sont mises à gérer ensemble.» (Entretien, 2008)

17. Mon soulignement. 
Peut-on imaginer une meilleure façon de contrôler (qu'il ne s'agit pas d'une fausse coopérative) que de faire en sorte que la gestion soit en effet assurée par les travailleurs associés? S'appuyer sur les travailleuses et travailleurs parait en tout cas plus approprié que de les dénoncer au ministère public pour réclamer leur transformation en entreprises déclarant des travailleurs formels, et les voir tout simplement fermées par l'entreprise donneuse d'ordres, sans que les syndicats ne se préoccupent de ce que vont devenir les travailleurs, comme cela s'est produit dans des régions du Nordeste brésilien (Lima, 2003).

Mais faire appel aux travailleurs des fausses coopératives parait irréaliste aux yeux d'interlocuteurs du syndicat des métallos de l'ABC pour qui - argument classique - ces travailleurs paraissent trop précaires, trop dispersés, etc., pour être organisés. En fait, ils ne considèrent pas les travailleurs et leurs mobilisations comme les leviers de la transformation sociale (ou comme des opportunités) mais comme les freins.

Le texte de référence des orientations dans l'économie solidaire adopté par la CUT en 1999 souligne ainsi combien les travailleurs et, en l'occurrence, les coopérateurs, ne sont pas capables par eux-mêmes de poursuivre la lutte contre les fausses coopératives en maintenant les objectifs solidaires sans l'intervention du syndicat et d'une législation appropriée - quand bien même ils seraient éduqués "pour décider démocratiquement d'opter pour la création de fonds dans les coopératives qui garantissent ces droits [des travailleurs formels] » (Magalhães et Todeschini, 2000: 21).

C'est la même analyse qui est reprise en 2008 par un interlocuteur du syndicat des métallos de l'ABC pour qui, oui, il faut fermer ces initiatives qui ne fournissent pas au moins le salaire minimum, car elles font le lit des fausses coopératives:

Je crois que ceux qui n'ont pas de viabilité économique, ce n'est pas en travaillant 18 heures par jour qu'ils vont y parvenir [...] parce que cela crée une situation artificielle de travail, où les personnes sont en train de se tuer pour travailler [...]. Il y a une erreur là-dedans: soit c'est le produit, ou le processus de travail ou la gestion. Donc moi, je ne peux pas au nom du coopérativisme permettre que les personnes s'appauvrissent chaque fois plus, travaillent chaque fois plus et que quelqu'un d'autre [que ces coopérateurs ou associés] gagne ainsi beaucoup d'argent. (Entretien, 2008)

Cette façon d'identifier les problèmes semble ignorer qu'il peut y avoir des initiatives qui ne décollent pas, non pas parce qu'elles vendent à des prix dérisoires en organisant le dumping social, mais à cause des nombreuses barrières existantes au développement d'activités, qui ont déjà été repérées pour les activités informelles (Lautier, 2006) et dont certaines se reproduisent dans l'économie solidaire. Ainsi de l'absence d'un capital de départ, du manque d'accès au crédit ou à une plus large clientèle, et du besoin de consommer immédiatement son revenu. Ces barrières ne relèvent pas d'erreurs humaines mais des inégalités socioéconomiques structurelles qui, pour être remises en causes, supposent des politiques publiques appropriées.

Or les politiques nationales d'appui à l'économie solidaire du gouvernement Lula sont restées résiduelles (Carvalho Barbosa, 2007) car elles n’ont justement visé que la gestion de la pauvreté. Globalement, les réformes structurelles n'ont pas suivi, que ce 
soit en termes de politiques macroéconomiques ou en termes de protection sociale et d'éducation, qui continuent à fonctionner de manière dualiste au Brésil. Les politiques menées par le gouvernement Lula, conseillé notamment par des présidents du syndicat des métallos de l'ABC (entretien 2008), ont ainsi conduit à des impasses ${ }^{18}$. Leur priorité était de soutenir la croissance et la création d'emplois formels par « une action plus efficace de la part de la justice du travail dans le contrôle et la répression des entreprises délinquantes» (Carleial, 2014: 70). Près de 18 millions d'emplois formels ont ainsi été créés entre 2000 et 2010. Mais ce décompte intègre par exemple ceux créés par les agences de travail temporaire (Guimaraes, 2011).

Voilà à quoi aboutit la version critique du développement élaborée par des intellectuels latino-américains (dont Quijano): on prend en compte la lutte contre les inégalités, mais en acceptant le paradigme dominant. Résultat: au lieu de voir la recrudescence de l'informalité comme l'expression d'une transformation des modes de régulation, au Sud comme au Nord, on la réduit aux emplois informels, eux-mêmes associés au sous-développement (Lesemann, 2014).

\section{LES CORRESPONDANCES AVEC LES PRATIQUES AUTOGESTION NAIRES}

\subsection{Naturalisation de l'économie et reproduction des divisions hiérarchisées du travail}

Les pratiques autogestionnaires ont un caractère formel dans les coopératives citées en référence par le syndicat des métallos de l'ABC, par Unitrabalho et par une partie de l'exécutif d'Unisol partageant leurs positionnements, dans les controverses. La gestion de ces coopératives est calquée sur le modèle des entreprises classiques, car c'est la façon d'atteindre une compétitivité économique dont les normes ne rompent pas avec celle de la logique marchande capitaliste. L'idée est explicitement défendue par l'un des responsables des trois coopératives citées le plus souvent par ces interlocuteurs (entretiens 2008) et par la littérature scientifique brésilienne sur le sujet (Marques, 2006):

Nous ne sommes pas contre le capitalisme. Nous ne voulons pas rivaliser avec lui, car on utilise le même outil: nous nous formons, nous nous habilitons, nous nous faisons certifier ISO, et tout cela pourquoi? [...] Ce que nous voulons, c'est disputer le marché. La seule différence avec le capitalisme, c'est la façon dont tu vas distribuer le revenu; c'est le contraire du capitalisme qui ne distribue rien, qui concentre tout le revenu. (Responsable de coopérative, 2008)

18. D’où les importantes manifestations de rue au Brésil en 2013, sous le gouvernement de Dilma Roussef qui, devant faire face à l'aggravation de la situation économique et à l'alourdissement de la dette, n'a plus les marges de manœuvre de Lula. À noter que parallèlement, les pauvres et en particulier les mères, se sont fortement mobilisés contre l'idée de restreindre la Bolsa familia, la principale mesure adoptée par Lula pour lutter contre la pauvreté et qui a permis à plus de 13 millions de ménages (chiffres 2011) de sortir de l'extrême pauvreté (Souza, 2013). 
Ces trois coopératives (une dans l'ABC, deux autour de Porto Alegre) sont des entreprises récupérées considérées comme ayant réussi, alors qu'elles ont «abandonné au nom du succès économique» quelques-unes des «caractéristiques importantes du travail autogéré» (Costa et Dagnino, 2009: 12). Même si les travailleurs sont propriétaires des moyens de production et se réunissent régulièrement en assemblée générale, les coopératives n'échappent pas à la reproduction des rapports sociaux inégaux qui se structurent autour des divisions du travail entre concepteurs et exécutants ou intellectuels et manuels (Marques, 2006; Costa et Dagnino, 2009; Rosenfield, 2007). L'organisation du travail et la répartition des tâches ne font même pas toujours l'objet de débats. Et quand c'est le cas, en général juste après la récupération de l'entreprise et sa transformation en coopérative (syndicaliste responsable de coopérative, 2008), les choix opérés aboutissent à ce qu'un petit noyau de personnes qualifiées ou de syndicalistes concentrent le pouvoir, pour de multiples raisons repérées par différents auteurs: le noyau de personnes qualifiées détient un savoir-faire technologique qu'elles ne partagent pas (Lima, 2008; Holzmann, 2012) ou le président de la coopérative est payé pour assumer les contacts avec les partenaires extérieurs (banques, élus locaux, organismes d'appui et de conseil-accompagnement) et se faire déléguer aux diverses rencontres des forums locaux et nationaux du FBES, si bien qu'il concentre un capital social irremplaçable (Holzmann, 2012). Enfin, les travailleurs peu qualifiés n'osent pas prendre la parole lors des assemblées générales. Il est même arrivé qu'ils en sortent pour reprendre le travail (Rosenfield, 2007).

Cette attitude de retrait face au projet autogestionnaire est attribuée à des caractéristiques individuelles par les syndicalistes ou des responsables de ces coopératives. Ils mettent ainsi en cause l'âge, la sclérose des routines, les réticences ou les peurs de se lancer dans l'aventure autogestionnaire. Il y aurait un «choc culturel» avec le passage d'une structure organisationnelle rigide et autoritaire, où les travailleurs avaient l'habitude de se conduire en exécutants, à une structure autogérée, qui réclame d'autres comportements (Guimarães, Korosue et Correa, 2006: 308). Les travailleurs voudraient maintenir les relations patron-salariés traditionnelles: "[ils] veulent que quelqu'un leur dise quoi faire: «Écoute, tu vas faire ceci, tu vas faire cela» et à la fin du mois, [ils] reçoivent leur argent. Donc, un type de personnes comme ça a du mal à changer, mais il y en a beaucoup» (Responsable de coopérative, 2008).

Cependant, des travaux mentionnent que des travailleurs-coopérateurs se montrent insatisfaits de constater que l'autogestion n'a pas remis en cause les hiérarchies existantes ni la parcellisation ou la fragmentation du travail, typique du taylorisme ${ }^{19}$. On pourrait dire qu'en réalité, il n'y a justement pas eu de choc culturel. Comme le souligne Méda (voir 1995, p. 161), l'appropriation collective des moyens de production ne fait pas disparaître le caractère aliénant du travail hétéronome, quand elle reste au service d'un objectif — la compétitivité — qui est à la source même

19. Faria (de), Mauricio S. (2005), Autogestão, Cooperativa, Econonomia Solidária: Avatares do Trabalho e do Capital, [tese de doutorado em sociologia Política], Florianopolis, UFSC. Cité par Marques (2006: 78). 
de l'exclusion des coopérateurs peu qualifiés; ceux-ci, maintenus dans une position d'exécutant, adoptent donc une position de «retrait» face au projet autogestionnaire (Rosenfied, 2007).

Le phénomène de retrait se retrouve d'ailleurs dans les coopératives qui ne sont pas issues d'entreprises récupérées, mais dont l'objectif est d'atteindre la compétitivité. Ainsi, à Diadema, dans une coopérative de catadores soutenus par le syndicat des métallos de l'ABC, où $90 \%$ des membres sont d'anciens métallos: le président, ancien syndicaliste qui a «fait les grèves ${ }^{20}$ », se plaint de ce qu'il est difficile de « conscientiser » à l'autogestion et de ce que les coopérateurs ont un rapport instrumental au projet: «Les membres ne voudraient que travailler et recevoir leur salaire; travailler et recevoir; travailler et recevoir. Ils viennent des entreprises alentour, ils ont une mentalité de subordonné.»

Dans ces coopératives, l'autogestion reste un projet idéologique extérieur à la finalité du travail. Cette conception est cohérente avec une vision naturalisée de l'économie informelle et d'un développement étapiste. Elle a d'ailleurs débouché dans l'ABC sur une politique classique de construction d'une chaine productive du recyclage, avec la décision d'incinérer une partie des déchets prise par la mairie de San Bernardo (autre ville de l'ABC) après l'élection en 2008 de Luis Marinho, ancien président du syndicat des métallos de l'ABC. Une opération technologiquement brillante, mais en réalité très coûteuse et ne faisant pas appel aux coopératives de catadores (Instituto Humanitas Unisinos, 2014), à laquelle la mairie a finalement renoncé devant la mobilisation des catadores non anciens métallos.

\subsection{La construction de l'implication des catadores dans la chaîne de recyclage du Rio Grande do Sul}

Les acteurs sociaux ayant contribué à la construction de la chaîne productive du recyclage dans le Rio Grande do Sul ont d'autres références de pratiques autogestionnaires en tête que les exemples cités ci-dessus. Pour eux, le défi n'est pas seulement d'arriver à être économiquement viable, mais à ce que les travailleurs s'impliquent et à ce que leurs aspirations soient prises en compte.

La coopérative Univens constitue à ce titre l'une de leurs références. Réussissant sans perdre son caractère autogestionnaire, elle pourrait passer pour l'exemple concret des théories utopistes de Marx, pour qui le travail est émancipateur et vecteur de lien social si le collectif de travailleurs produit pour satisfaire des besoins sociaux décidés collectivement (Méda, 1995). À Univens, l'organisation du travail a d'ailleurs été pensée de façon à diviser les tâches sans les parcelliser, pour que la finalité de l'acte reste présente. La répartition des responsabilités a aussi été organisée de façon à éviter la centralisation du pouvoir entre quelques mains: "Le conseil d'administration ne décide de rien. Il prépare les sujets mais ne décide pas» (entretien 2008). Les tâches

20. Il fait référence aux grandes grèves de la fin des années 1970 et début des années 1980 contre la dictature. 
internes et les relations avec les activités locales et nationales du mouvement de l'économie solidaire tournent entre les 26 associés [ 25 femmes et un homme] : «Nous avons fait une liste de responsabilités [...]. Le café... L'homme associé a les mêmes responsabilités que les femmes» (ibid.). Par ailleurs, personne, pas même la présidente de la coopérative, n'est financièrement dédommagée pour sa participation aux diverses rencontres du FBES: «Quand on va à une réunion, si elle est dans une autre ville, on peut perdre un ou deux jours de production» (ibid.).

Parallèlement, la coopérative participe à des activités communautaires et des mouvements sociaux, pour s'intégrer «aux autres luttes» (ibid.), en créant les conditions, là encore, pour que tout le monde participe. Cela nourrit les échanges entre les travailleurs des coopératives ou associations autogérées et les communautés (locales ou militantes) pour «savoir quoi produire, pour qui [et] comment produire», comme le résume un responsable du syndicat des métallos de Porto Alegre, pour qui le thème de l'autogestion et celui de l'autonomie doivent aller de pair.

Or, ce qui paraît important dans cette participation d'Univens aux activités communautaires et aux mouvements sociaux, c'est qu'elle contribue à ce que ses membres soient amenées à

comprendre que ce qu'elles font est en relation avec le monde, avec la société qui est en train de se faire [...]. Il y a des jours ici où tout le monde participe à la discussion, avec les machines à coudre placées côte à côte. Et des fois, ce sont des sujets bien chouettes, sur la politique, tout ça. Alors je crois que c'est cela qui fait que les personnes se développent et participent $^{21}$. (Entretien, 2008)

Voilà un aspect qui mérite qu'on l'examine. Car il montre qu'à Univens, dont l'histoire est marquée par le budget participatif de Porto Alegre, on n'y fait pas que travailler. Si rapport autonome il y a au travail, c'est sans doute parce que le lien social ne repose justement pas que sur le travail marchandisé, mais sur diverses activités, y compris politiques au sens large, qui permettent aussi aux individus de trouver des moyens de s'affirmer dans le cadre d'une production collective ${ }^{22}$.

Tout l'enjeu et la difficulté des mobilisations de l'économie solidaire est d'arriver à maintenir l'implication des membres dans la vie de la coopérative: la pente naturelle est de se centrer sur le travail et son efficacité (ou de se mettre en retrait), puisque la viabilité des coopératives passe par le fait de s'assurer un débouché sur un marché régi par la recherche du profit. Ceci limite le contrôle que les associés peuvent exercer sur la gestion (Guimaraes, Korosue e Correa, 2006) et rend justement difficile leur implication, si l'on ne discute pas explicitement des solidarités possibles, en créant des espaces de débat au sein de la coopérative et au-delà (Sarria Icaza, 2008).

21. Notre soulignement.

22. Voir Méda (1995) sur la critique faite à Marx de continuer à considérer le travail à la fois comme un moyen de produire des richesses et comme le vecteur du lien social. Voir aussi Galerand et Kergoat (2014) sur la transformation des rapports sociaux à partir du fait de considérer toutes les activités, rémunérées ou non, privées ou publiques, comme contribuant à la production du «vivre-ensemble». 
Complexes, délicates, ces réflexion et action collective sont toutefois explicitement assumées à travers la recherche de méthodologie d'accompagnement des initiatives et de validation de stratégies par une partie des empreendimentos de l'économie solidaire et des organismes communautaires accompagnateurs (responsable, organisme communautaire, 2008). Les syndicats qui l'assument sont en général en conflit avec l'orientation majoritaire de la CUT dans l'économie solidaire.

Ceci explique la forme d'implication du syndicat des métallurgistes de Porto Alegre, qui a créé en 2003, à la suite d'un vote unanime en assemblée générale, un fonds de soutien à tous les types d'initiative de l'économie solidaire, et pas seulement aux coopératives compétitives, pour autant que ces groupes tendent à respecter un «format». Il repose sur trois critères: la division juste des produits du labeur; la pratique réelle de l'autogestion — c'est-à-dire le fait que «tous les travailleurs donnent leur opinion sur le processus productif» — ; et la démocratie — «c'est-à-dire le fait qu'il existe bien des règles permettant l'existence d'un espace où tous peuvent donner leur opinion» (responsable syndical, 2008). On n'est donc pas seulement dans la tenue formelle d'assemblée générale, il s'agit de rendre effectifs l'implication et la prise de parole, comme c'est le cas à Univens que le syndicat a soutenu dès sa création en 1996.

En 2008, les empreendimentos soutenus par le fonds avaient commencé à échanger sur la façon de mieux accompagner les initiatives dans l'économie solidaire. Parallèlement, le syndicat des métallos était engagé dans la relance d'une tentative de construction d'une chaîne productive du recyclage avec l'objectif explicite non seulement de développer son industrialisation, mais aussi d'arriver à créer les conditions de l'engagement dans cette chaîne des catadores, y compris des groupes fragiles car composés de moradores da rua (littéralement habitants des rues), dont certains dorment sur les déchets. Il collaborait à cette fin avec les organismes communautaires déjà impliqués et avait mobilisé divers organismes proches de la CUT ainsi qu'une école technique régionale spécialisée dans le recyclage du plastique: «Nous sommes en train d'étudier le sujet avec les groupes de tri. Eux aussi doivent donner leur avis. Alors, on est en train d'aider à reconstruire la fédération des recycleurs [fédération locale du MNCR] ; les groupes de tri ont l'appui du syndicat, de la CUT, de plusieurs organisations» (ibid., 2008).

C'est l'existence du fonds de soutien qui avait conduit des groupes de catadores à frapper à la porte du syndicat pour pouvoir s'acheter une balance... Depuis, la fédération du MNCR sur le Rio Grande do Sul est devenue une adhérente d'Unisol. Elle s'implique dans l'organisation des catadores des rues et pour généraliser le recours aux coopératives de catadores par les municipalités, dans le cadre de la Politique Nationale des Résidus Solides (Instituto Humanitas Unisinos, 2014).

\section{CONCLUSION}

Cet article a permis de mettre en évidence comment les stratégies syndicales et politiques sont reliées aux façons d'analyser la recrudescence de l'économie informelle et le rôle des mobilisations de travailleurs (comme leviers ou freins) dans le changement 
social. L'article montre comment les façons d'analyser sont reliées à la reconnaissance, ou non, dans l'arène publique de l'apport des mobilisations de travailleuses et travailleurs dans l'économie solidaire, et comment cette reconnaissance est interdépendante des conditions selon lesquelles les acteurs sociaux et politiques entrent en interaction avec ces travailleurs mobilisés - notamment à travers les budgets participatifs et les pratiques autogestionnaires dans les coopératives ou entreprises récupérées.

Si l'informalité en tant que catégorie peut nous renseigner sur l'évolution de nos sociétés contemporaines (Lesemann, 2015), les mobilisations dans l'économie solidaire rendent visibles les liens entre, d'une part, les choix de production et son organisation et, d'autre part, les façons dont les travailleuses et travailleurs transforment leur rapport au travail quand le lieu de travail n'est plus seulement un lieu de production mais aussi de débats sur leurs besoins et aspirations. Elles montrent ainsi ce que peuvent être des luttes pour l'égalité qui dépassent les formulations en termes de salaires, de durée du travail et d'avantages attachés à l'emploi conventionnel (voir Portes et al., 1989: 309-310), et qui, en outre, apportent des réponses hic et nunc à la nécessité de dépasser un modèle de développement reposant sur l'idéologie de la croissance «illimitée» (Silver, 2003: 178) en contradiction avec la protection de l'environnement.

Finalement, cet article fait ressortir que, pour percevoir la capacité des mobilisations de travailleurs au cœur des multidominations de produire des connaissances sur le monde, il s'avère nécessaire, d'une part, d'élargir la notion de travail et celle de travailleur, habituellement restreinte au travailleur formel ou standard, ce qui bloque la reconstruction de solidarités (Lindell, 2011; Kenny, 2011) et, d'autre part, et corollairement, de remettre en cause la prédominance des cadres d'analyse issus de la société salariale, qui n'accordent un rôle transformateur qu'aux luttes du mouvement ouvrier organisé dans les syndicats ou se déroulant dans les lieux de travail classiques.

\section{RÉSUMÉ}

Rassemblant au Brésil toute une partie des travailleurs informels, l'économie solidaire est un sujet controversé parmi les acteurs syndicaux et politiques. Est-elle un lieu d'insertion des travailleurs, et (surtout) travailleuses, pauvres ou informels qui sont rejetés par un marché nécessairement compétitif? Ou les différentes mobilisations, et certaines des pratiques autogestionnaires de ces travailleurs dits marginalisés, constituent-elles des luttes pour l'égalité et la justice sociale qui dépassent les revendications (en termes de salaires, de durée du travail, etc.) issues du compromis de la société salariale - compromis qui n'a jamais eu vocation à couvrir plus qu'une minorité de travailleurs dans le monde? Étayé par des entretiens approfondis menés auprès de responsables communautaires, syndicaux et politiques impliqués dans l'économie solidaire au Brésil, l'article met ainsi en lumière les relations entre leurs choix stratégiques et les façons d'analyser le problème de la remontée du travail informel. Les controverses suscitées sont révélatrices de conceptions plus globales sur l'économie et la place des mobilisations de travailleurs dans le changement social.

Mots clés: travail informel, problème public, identité collective, rapports sociaux, stratégies syndicales 


\begin{abstract}
Bringing together a whole section of informal workers in Brazil, the solidarity economy is a controversial topic among union and political actors. Is it a space for the integration of workers, especially poor or informal women workers who are rejected by a market that is necessarily competitive? Where different mobilizations, and certain self-organizing practices of these socalled marginalized workers are struggles for equality and social justice that go beyond specific demands (in terms of salaries, working hours, etc.) derived from the pledge of a wage-based society - a pledge that was never meant to cover more than a minority of the world's workers? Supported by in-depth conversations with community, union and political leaders in Brazil's solidarity economy sector, the article sheds light on the relations between the strategies they have chosen and different ways of analyzing the issue of the resurgence of informal work. The controversies raised reveal more global visions of the economy and the place of worker mobilizations in social change.
\end{abstract}

Key words: informal work, public issues, collective identity, social relations, labour union strategies.

\title{
RESUMEN
}

La economía solidaria en Brasil, que agrupa una gran parte de los trabajadores informales, es un tema controvertido entre los actores sindicales y políticos. ¿Es este un lugar de inserción de los trabajadores y (sobre todo) de trabajadoras pobres o informales, expulsadas del mercado, necesariamente competitivo? ¿O las diferentes movilizaciones y ciertas prácticas autogestionarias de estos trabajadores llamados marginalizados constituyen luchas por la igualdad y la justicia social que van más allá de las reivindicaciones (en términos de salarios, duración del trabajo, etc.) provenientes del compromiso de la sociedad salarial —compromiso que nunca ha tenido la vocación de cubrir más que a una minoría de trabajadores en el mundo? Apoyándose en diferentes entrevistas exhaustivas realizadas a líderes comunitarios, sindicales y políticos implicados en la economía solidaria de Brasil, este artículo destaca las relaciones entre sus opciones estratégicas y diferentes maneras de analizar el problema del auge del trabajo informal. Las controversias suscitadas revelan concepciones más globales acerca de la economía y del lugar que ocupan las movilizaciones de los trabajadores en el cambio social.

Palabras clave: trabajo informal, problema público, identidad colectiva, relaciones sociales, estrategias sindicales

\section{BIBLIOGRAPHIE}

Agarwala, R. (2009), «An economic sociology of informal work: The case of India», Economic Sociology of Work, Research in the Sociology of Work, vol. 18, p. 315-342.

Agarwala, R. (2013), Informal Labor, Formal Politics and Dignified Discontent in India, Cambridge: Cambridge University Press.

Armstrong, E. et M. Bernstein (2008), «Culture, Power, and Institutions: A Multi-Institutional Politics Approach to Social Movements», Sociological Theory, vol. 26, n 1, p. 74-99.

Bernstein, M. (2008), «Afterword: The Analytic Dimensions of Identity: A Political Framework», in J. Reger, R. Einwohner et D. Myers, Identity Work in Social Movements, Minneapolis, University of Minnesota Press, p. 277-302. 
Baḯcchi, G. (2003), «Emergent Public Spheres: Talking Politics in Participatory Governance», American Sociological Review, vol. 68, $\mathrm{n}^{\circ} 1$, p. 52-74.

Barcellos, J. A. et R. E. V. Beltrão (2000), «Institução Comunitaria de Credito Portosol: Construindo uma economia solidária», in P. Singer et A. R. de Souza (dir..), A economia solidária no Brasil. A Autogestão como resposta ao desemprego, São Paulo: Contexto, p. 161-192.

Bieler, A (2014), «Transnational Labour Solidarity in (the) Crisis», Global Labour Journal, vol. 5, n² 2 , p. 114-133.

Brenner, N. (1999), «Beyond state-centrism? Space, territoriality, and geographical scale in globalization studies", Theory and Society, $\mathrm{n}^{\circ} 28$, p. 39-78.

Carleial, L. M. da F. (2014), «Sous-développement et marché du travail au Brésil», in P. J. Ulysse, F. Lesemann et F. J. Pires de Sousa, Les travailleurs pauvres. Précarisation du marché du travail, érosion des protections sociales et initiatives citoyennes, Québec, Presses de l'Université du Québec, p. $45-88$.

Carvalho Barbosa, R. N. (2007), "Trabalho informal e políticas publicas para geraçao de renda», XIII Congresso Brasileiro de sociología do trabalho, 29 de maio a 01 de junho de 2007, Recife (Pe), UFPe.

Chun, J. J. (2009), Organizing at the Margins. The Symbolic Politics of Labor in South Korea and the United States, Ithaca, NY, Cornell University Press.

Costa, E. H. D. S. et R. P. Dagnino (2009), «A Experiência da Fábrica Recuperada Uniforja: Autogestão ou Heterogestão?», in Congresso Brasileiro de Sociologia, 2009, Rio de Janeiro, UFRJ.

Croucher, R. (2007), «Organizing the Informal Economy: Results and Prospects. The Case of Ghana in Comparative Perspective», in G. Wood et C. Brewster (dir.), Industrial relations in Africa, Basingstoke, Palgrave Macmillan, p. 209-218.

Delphy, C. (2001 [1998]). L'ennemi principal, tome 1. Économie politique du patriarcat. Paris, Syllepse.

Diani, M. (2003), «Introduction: Social Movements, Contentious Actions, and Social Networks: From Metaphor to Substance?», in D. Mario et D. McAdam (dir.), Social Movements and Networks: Relational approaches to collective action, New York, Oxford University Press, p. 1-18.

Ferraz, M. (2005), Da cidadania salarial à agência de desenvolvimento solidário. O Sindicalismo-CUT e os desafios para inventar uma nova cidadania. [Tese] apresentada ao Programa de Pós-Graduação em Sociologia da Faculdade de Filosofia, Letras e Ciências Humanas da Universidade de São Paulo.

Fernandez-Kelly, P. (2006), "Introduction», in Fernandez-Kelly P. et J. Shefner (dir.), Out of the shadows: Political action and the informal economy in Latin America, University Park, The Pennsylvania State University Press, p. 1-22.

Flyvbjerg, B. (2006), «Five Misunderstandings about Case-Study Research», Qualitative Inquiry, vol. 12, $\mathrm{n}^{\circ} 2, \mathrm{p} .219-245$.

Gadrey, J. (2002), «Les conventions de service», dans J. Gadrey et P. Zarifian, L'Émergence d'un modèle de service: enjeux et réalités, Paris, Éditions Liaison, p. 157-162.

Gaiger, L. I. (2000), «Os caminhos da economia solidária no Rio Grande do Sul», in P. Singer et A. R. de Souza (dir.), A economia solidária no Brasil. A Autogestão como resposta ao desemprego, São Paulo, Contexto, p. 267-288.

Galerand, E. et Kergoat, D. (2014), «Les apports de la sociologie du genre à la critique du travail», $L a$ nouvelle revue du travail, $\mathrm{n}^{\circ} 4$, [en ligne] consulté le 2 juin 2014: http://nrt.revues.org/1533

Georges, I. (2011), «Une faible amélioration de la représentation collective des travailleurs informels », Chronique internationale de l'IRES, n 130 , p. 42-54.

Guimarães, V. N., Korosue A. e F. Z. M. Correa (2006), «Empreendimentos autogeridos em Santa Catarina: uma alternativa democratica a produção ", in V. Piccinini et al. (dir.), O mosaico do travalho na sociedade contemporanea, persistências e inovaçoes, Porto Alegre, Editora da UFRGS, p. 293-324.

Guimarães, N. A. (2011), «As novas configurações do trabalho formal. Algumas reflexões a luz do caso de São Paulo», Encontro annual da ANPOCS. Caxambu: ANPOCS, 24 à 28 Outubro. En ligne (imprimé le 2 février 2012) : www.anpocs.org/portal/index.php? option=com_docman\&task=doc_ view\&gid $=1249 \&$ Itemid $=353$ 
Goodwin, J. et J. M. JAspers (2004), «Caught in a Winding, Snarling, Vine: The Structural Bias of Political Process Theory», in J. Goodwin et J. M. Jasper (dir.), Retinking Social Movements: Structure, Meaning, and Emotion, Lanham, Rowman \& Littlefield Publishers.

Holzmann, L. (2012) «Empreendimentos solidarios. Autonomia ou tutela?», in I. Georges et M. P. de Leite, Novas configurações do trabalho e economia solidária, São Paulo, Annablume.

INSTITUTO HUMANITAS UNISINOS (2014). «Reciclagem de resíduos sólidos: a propaganda é bonita, mas o processo explora os catadores», Entrevista especial com Alex Cardoso. [En ligne] : www.ihu. unisinos.br/entrevistas/532579-reciclagem-de-residuos-solidos-a-propaganda-e-bonita-mas-oprocesso-explora-os-catadores-entrevista-especial-com-alex-cardoso

Jenson, J., M. Mendell, C. Martin et D. Renard (1999), «Les mots pour le dire, les mots pour le faire: le nouveau vocabulaire du social», Lien social et Politiques, RIAC - 41, p. 5-12.

Kenny, B. (2011), «Reconstructing the Political Mall? Committees and South African Precarious Retail Workers », LABOUR, Capital and Society, TRAVAIL, capital et société, Formal/Informal Divide, Rupture entre les secteurs formel/Informel, vol. 44, $\mathrm{n}^{\circ}$ 1, p. 44-69.

Kergoat, D. (2009), «Dynamique et consubstantialité des rapports sociaux», dans E. Dorlin, Sexe, race, classe, pour une épistémologie de la domination, PARIS, PUF, p. 111-125.

LAutier, B. (2003), «Les limites de l'économie informelle comme alternative à la mondialisation libérale», Revue du Mauss, vol. 1, n² 21, p. 198-214.

LAutier, B. (2006), L'économie informelle dans le tiers-monde, Paris, la Découverte.

Lechat, N. (2004), Trajetórias intelectuais e o campo da Economia Solidária no Brasil. [Tesis de Doctorado], Universidad Estadual de Campinas.

Leite, M. P. de (2009a), "O trabalho e suas reconfigurações: conceitos e realidades», in A. M. C. Araujo et M. de P. Leite (dir.), O trabalho reconfigurado: ensaios sobre Brasil e México, São Paulo, Annablume.

Leite, M. P. de (2009b), "A economia solidária e o trabalho associativo: teorias e realidades», Revista Brasileira de Ciencias Sociais, vol. 24, n 69, p. 31-51.

Lesemann, F. (2015), “"Travail informel”, informalité et "société salariale”. Centrer la réflexion relative aux transformations du travail et des sociétés contemporaines sur le " travail informel " et l'informalité plutôt que sur la société salariale», dans M. D’Amours, S. A. Soussi et D.-G. Tremblay (dir.), Repenser le travail. Des concepts nouveaux pour des réalités transformées, Québec, Presses de l'Université du Québec, p. 247-284.

LESEMANN, F. (2014), "L'informalité et le travail informel comme analyseurs de la transformation de l'activité de production et du rôle des États. Une comparaison Nord-Sud», in P.J. Ulysse, F. Lesemann, F. J. Pires de Sousa, Les travailleurs pauvres. Précarisation du marché du travail, érosion des protections sociales et initiatives citoyennes, Québec, Presses de l'Université du Québec, p.89-108.

Lima J. C. (2003), «O trabalho autogestionario em cooperativas de produção. A retomada de um velho paradigma», ANPOCS, Caxambu, 21-25 de outubro de 2003.

Lima J. C. (2008), «Reestruturação industrial, desemprego e autogestão: as cooperativas do Vale do Sinos », revista Sociologias, Porto Alegre, vol. 10, n²1, p. 1-27.

Lindell, I. (2010), «Informality and Collective Organising: Identities, alliances and transnational activism in Africa», Third World Quaterly, vol. 31, n² 2, p. 207-222.

Lindell, I. (2011), «Organizing across the formal-informal worker constituencies/Faire le pont entre les secteurs formel et informel», LABOUR, Capital and Society, Formal/Informal Divide, Rupture entre les secteurs formel/informel, TRAVAIL, capital etsSociété, vol. 44, n 1, p. 2-17.

Magalhaes, R. S. et R. Todeschini (2000), Sindicalismo e economia solidária: reflexões sobre o projeto da CUT», in P. Singer et A. R. de Souza (dir.), A economia solidária no Brasil. A Autogestão como resposta ao desemprego, São Paulo: Contexto, p. 135-160.

Marques, P. L. (2006), «Trabalho Emancipado. Empresas recuperadas pelos trabalhadores: A experiença autogestionaria de metalúrgicos gauchos », Programa de pos graduação em sociologia, Porto Alegre, UFRGS.

MédA, D. (1995), Le travail, une valeur en voie de disparition, Paris, Aubier-Flammarion. 
Mcadam, D. (2003), «Beyond Structural Analysis: Toward a More Dynamic Understanding of Social Movements", dans M. Diani et D. McAdam (dir.), Social Movements and Networks: Relational Approaches to Collective Action, New York, Oxford University Press, p. 281-298.

Melucci, A. (1996), Challenging Codes. Collective Action in the Information Age, Cambridge, Cambridge University Press.

Paoli, M. C. (2000), «Institution et destitution des droits sociaux au Brésil», Mana n 7, Presses universitaires de Caen, p. 27-44.

Portes, A., Castells, M. et L. A. Benton (1989), «The Policy Implications of Informality», in Portes, A., Castells, M. et L. A. Benton, The Informal Economy. Studies in Advanced and Less Developed Countries, Baltimore, The Johns Hopkins University Press, p. 298-311.

Ramalho, J. R. (1999), «Restructuring of Labour and Trade Union Responses in Brazil», in R. MuncK et P. WATERMAN (dir.), Labour worldwide in the era of globalization. Alternative union models in the new world order, New York, St. Martin's Press.

Ramalho, J. R. et M. A. Santana (2003), «Trabalhadores, sindicatos e a nova questão social», in M. A. Santanaet J. R. Ramalho (dir..), Além da fabrica. Trabalhadores, sindicatos e a nova questão social, São Paulo, Boitempo Editorial, p. 11-43.

Rosenfield, C. L. (2007), «A autogestão e a nova questão social: repensando a relação individuosociedade», in Lima, J. (dir.), Ligações perigosas. Trabalho Flexível e Trabalho Associado, São Paulo, Annablume, p. 93-126.

Sarria Icaza, A. M. (2005). «Politiques publiques et économie solidaire au Rio Grande do Sul», in Action publique et économie solidaire. Une perspective internationale, J.-L. LAVILLE (dir.), J.-P. MAGNen, G. C. de França Filho et A. Medeiros, Toulouse (France), érès, p. 243-258.

SARria IcAZA, A. M. (2008), Economia solidária, accion colectiva e espacio publico en el sur de Brasil, Thèse de doctorat, Université catholique de Louvain, Faculté des sciences économiques et sociales.

Silver, B. J. (2003), Forces of Labor. Workers movements and globalization since 1870, New York, Cambridge University Press.

SINGER, P. (2002), «A recente ressurreição da economia solidária no Brasil», in B. de SousA SANTos (dir.), Produzir para viver: os caminhos da produção não capitalista, Rio de Janeiro, Editora Civilização Brasileira, p. 81-130.

SouZA, A. R (2009), «A construção social e a investigação cientifica da economia solidária», VI ENEDS - Campinas, SP, Brasil, 17 e 18 de Setembro de 2009.

Sousa, F. J. de P. (2013), «Brésil, une interprétation des récentes manifestations de rue», Chronique internationale de l'IRES, $\mathrm{n}^{\circ} 142$, p. 60-68.

Surel, Y. (1998), «Idées, intérêts, institutions dans l'analyse des politiques publiques», Pouvoirs, nº 87, p. 161-178.

Stroовалтs, M. (1993), Savoir-faire et compétences au travail. Une sociologie de la fabrication des aptitudes, Bruxelles, Éditions de l'Université de Bruxelles.

Yеrоснешsкі, С. (2015). La crise du syndicalisme comme crise de la représentation des travailleurs. Une étude de cas de l'évolution des identités collectives dans l'économie solidaire au Brésil, Thèse de doctorat, Montréal, Université de Montréal. 